\title{
Synthesis and characterization of a nanostructured porous silicon/carbon dot-hybrid for orthogonal molecular detection
}

\author{
Naama Massad-Ivanir ${ }^{1,5}$, Susanta Kumar Bhunia ${ }^{2,5}$, Nitzan Raz ${ }^{1}$, Ester Segal ${ }^{1,3}$ and Raz Jelinek ${ }^{2,4}$ \\ A new hybrid guest-host material consisting of a Fabry-Pérot porous silicon (PSi) thin film, a nanostructured high surface-area \\ matrix, and encapsulated fluorescent carbon quantum dots (C-dots) is described. The hybrid is synthesized by a facile in situ \\ pyrolysis treatment of the carbonaceous precursor incorporated within the nanoscale pores of the inorganic host. The effects of \\ nanoconfinement on the integrity of the C-dots and their optical properties are characterized. We show that the resulting hybrid \\ allows for label-free optical detection of target molecules using two orthogonal modalities, that is, the white-light reflectivity of \\ the PSi matrix and the fluorescence of the confined C-dots, and these two signals can be observed and collected simultaneously. \\ The resulting hybrid system exhibits superior sensing performance in comparison with that of the individual components. \\ Notably, we demonstrate that the confined C-dots exhibit greater sensitivity toward various analytes as well as an improved linear \\ response, thus providing evidence of the impact of the host nanoscale porous scaffold on the optical properties of the C-dots. \\ Moreover, we show that this orthogonal detection scheme increases the dynamic range of the sensor and minimizes false- \\ negative results.
}

NPG Asia Materials (2018) 10, e463; doi:10.1038/am.2017.233; published online 12 January 2018

\section{INTRODUCTION}

Carbon dots (denoted C-dots) are recently synthesized quasi-spherical carbonaceous nanoparticles ( $<10 \mathrm{~nm}$ average size) usually comprising graphitic cores surrounded by various surface functional units. ${ }^{1} \mathrm{C}$-dots have attracted significant interest because of their unique structural and photophysical properties and applications in diverse fields. ${ }^{2,3}$ Specifically, C-dots exhibit broad excitation-dependent fluorescence emissions (for example, multiple colors), bright luminescence and low photobleaching, which are beneficial for sensing applications. ${ }^{4-6}$ In particular, C-dots constitute a useful analytical platform because of their biocompatibility and potentially lower cytotoxicity compared with conventional semiconductor quantum dots (QDs). ${ }^{1}$ Moreover, C-dots can be synthesized from diverse inexpensive and readily available reagents through simple procedures, enabling straightforward modulation of their structural properties. ${ }^{4,7}$ Importantly, the fluorescence properties of C-dots are highly sensitive to their environments, and this feature has provided opportunities for various sensing applications. ${ }^{6,8}$ C-dot-based sensors have several advantages: (1) they exhibit high sensitivity toward various targets; (2) the sensing method is usually simple and free of reagents; and (3) the change in their fluorescence signal is very fast, which enables a very short response time. ${ }^{9-12}$
Porous silicon (PSi) and oxidized PSi $\left(\mathrm{PSiO}_{2}\right)$ have emerged in recent years as attractive and versatile nanomaterials for sensing applications. ${ }^{13,14} \mathrm{PSi}$ exhibits attractive, tunable properties that contribute to its use as a biosensor. Specifically, diverse PSi nano-architectures can be readily fabricated by electrochemical anodization; these PSi nanostructures exhibit high surface areas (up to $800 \mathrm{~m}^{2} \mathrm{~g}^{-1}$ ) and can be functionalized by a wide range of chemical and biological species. Importantly, white light reflectance-based interferometry constitutes a robust optical transduction method in PSi systems, making them powerful label-free detectors for various biomolecules. ${ }^{15-17}$ However, PSi biosensors suffer from practical limitations related to the single-mode optical sensing mechanism, which mainly include poor sensitivity (typical limit of detection in the micromolar range ${ }^{14,18}$ ) and lack of selectivity (for example, distinguishing between different analytes and the detection of more than one target analyte at once $\left.{ }^{14,19}\right)$. Extensive research efforts have been directed toward enhancing the sensing properties of PSi-based systems. ${ }^{20-22}$ Experimental approaches have usually involved different passivation chemical reactions or the design of complex optical devices. A recent strategy is the use of dual-mode transduction to enhance the biosensor sensitivity and expand the ability to detect

\footnotetext{
${ }^{1}$ Department of Biotechnology and Food Engineering, Technion-Israel Institute of Technology, Haifa, Israel; ${ }^{2}$ Department of Chemistry, Ben Gurion University of the Negev, Beer Sheva, Israel; ${ }^{3}$ The Russell Berrie Nanotechnology Institute, Technion—Israel Institute of Technology, Haifa, Israel and ${ }^{4}$ Ilse Katz Institute for Nanotechnology, Ben Gurion University of the Negev, Beer Sheva, Israel.

${ }^{5}$ These authors contributed Equally to this work.

Correspondence: Professor E Segal, Department of Biotechnology and Food Engineering, Technion—Israel Institute of Technology, Technion City, Haifa 3200003, Israel. E-mail: esegal@technion.ac.il

or Professor R Jelinek, Department of Chemistry, Ben Gurion University of the Negev, 1 Ben Gurion Avenue, Beer Sheva 8410501, Israel.

E-mail: razj@bgu.ac.il

Received 4 September 2017; revised 17 October 2017; accepted 12 November 2017
} 
several targets simultaneously. Recent work by the Weiss group on a Psi-nanostructured host containing QD-labeled biomolecules and colloidal gold nanoparticles demonstrated the potential of PSi-nanoparticle hybrids in dual-mode-sensing applications. ${ }^{23,24}$ Other studies have demonstrated the luminescence enhancement of the fluorophore molecules of QDs co-embedded within PSi host matrixes. $^{25,26}$

Herein, we report the fabrication of a bimodal sensing platform comprising C-dots integrated within a $\mathrm{PSiO}_{2}$ host matrix. Importantly, the $\mathrm{PSiO}_{2} / \mathrm{C}$-dot hybrid was constructed through a facile and robust single-step synthetic scheme in which the carbon precursor was incorporated into the $\mathrm{PSiO}_{2}$ matrix, and subsequent heating generated the encapsulated C-dots. The resulting hybrid $\mathrm{PSiO}_{2} / \mathrm{C}$-dot material enabled the orthogonal, bimodal sensing of biomolecules via modulation of both the optical reflectance associated with the $\mathrm{PSiO}_{2}$ matrix and the fluorescence of the C-dots. As a proof-of-concept, we applied the new system to the optical/fluorescence sensing of trypsin and adenosine triphosphate (ATP). The resulting hybrid system exhibited superior sensing performance in comparison to the individual components.

\section{MATERIALS AND METHODS}

\section{Materials}

Single-side polished and heavily doped p-type $\mathrm{Si}$ wafers $(0.0009 \Omega \mathrm{cm}$ resistivity, 〈100〉 oriented, B-doped) were purchased from Sil'tronix Silicon Technologies, Archamps, France. Aqueous HF (48\%) and absolute ethanol were supplied by Merck, Darmstadt, Germany. p-Phenylenediamine was purchased from Alfa-Aesar, Heysham, England. Trypsin, 2'-deoxyadenosine $5^{\prime}$-triphosphate disodium salt (ATP) and all buffer salts were purchased from Sigma-Aldrich Chemicals, Jerusalem, Israel. Phosphate-buffered saline at $\mathrm{pH}=$ 7.4 was prepared by dissolving $50 \mathrm{mM} \mathrm{Na} 2 \mathrm{HPO}_{4}, 17 \mathrm{mM} \mathrm{NaH}_{2} \mathrm{PO}_{4}$ and $68 \mathrm{mM} \mathrm{NaCl}$ in Milli-Q water $(18.2 \mathrm{~m} \Omega \mathrm{cm})$.

\section{Preparation of $\mathrm{PSiO}_{2}$}

Si wafers were anodized in a two-step process, and the conditions were optimized to produce a highly porous nanostructure with pores that were large enough to accommodate the C-dots $(>60 \mathrm{~nm})$. First, a sacrificial layer was etched in a 3:1 (v/v) solution of aqueous HF $(48 \%)$ and ethanol $(99.9 \%)$ for $30 \mathrm{~s}$ at a constant current density of $300 \mathrm{~mA} \mathrm{~cm}^{-2}$ in a custom-made Teflonetching cell; a platinum ring was used as the counter electrode. Next, the freshly etched porous layer was dissolved in a solution of $0.1 \mathrm{M} \mathrm{NaOH}$, and a subsequent etching step was performed under the above-mentioned conditions. After each step, the silicon surface was thoroughly rinsed with ethanol and dried under a stream of nitrogen. Finally, the PSi samples were thermally oxidized in a tube furnace (Thermo Scientific, Lindberg/Blue M $1200{ }^{\circ} \mathrm{C}$ SplitHinge, Waltham, MA, USA) at $800^{\circ} \mathrm{C}$ for $1 \mathrm{~h}$ in the ambient air to create a $\mathrm{PSiO}_{2}$ matrix. The oxidation process chemically stabilized the porous layer and created a hydrophilic surface that enabled a wide repertoire of chemical and biological modifications. ${ }^{14}$

\section{Characterization of $\mathrm{PSiO}_{2}$ films}

The structural properties, that is, thickness and porosity, of the $\mathrm{PSiO}_{2}$ layer were characterized by high-resolution scanning electron microscopy (HRSEM), gravimetric analysis (for porosity) and the spectroscopic liquid infiltration method, as previously described. ${ }^{27,28}$ The resulting porous layers were $\sim 5 \mu \mathrm{m}$ thick, and the calculated porosity was $\sim 80 \%{ }^{28}$

\section{In situ synthesis of C-dots within $\mathrm{PSiO}_{2}$}

The incorporation of C-dots in the porous nanostructure was achieved by in situ synthesis within the pores. A C-dot precursor solution $\left(25 \mathrm{mg} \mathrm{ml}^{-1}\right.$ p-phenylenediamine aqueous solution) was introduced onto the $\mathrm{PSiO}_{2}$ upper surface and allowed to infiltrate the pores, followed by evaporation of the water and mild, slow heating in an oven $\left(180^{\circ} \mathrm{C}, 12 \mathrm{~h}\right)$.

\section{Synthesis of 'free' carbon dots}

Preparation of 'free' C-dots was achieved by dissolving $100 \mathrm{mg}$ of p-phenylenediamine in $10 \mathrm{ml}$ of ethanol followed by mild, slow heating in a Teflon-lined autoclave chamber $\left(180^{\circ} \mathrm{C}, 12 \mathrm{~h}\right)$. The resulting C-dot solution was mixed with $10 \mathrm{ml}$ of distilled water, and the ethanol was removed from the mixture by rotary evaporation. The resulting aqueous C-dot solution was used for further experiments.

\section{Scanning electron microscopy}

HRSEM (Ultra Plus, Carl Zeiss, Oberkochen, Germany) studies of the neat $\mathrm{PSiO}_{2}$ samples and the $\mathrm{PSiO}_{2} / \mathrm{C}$-dot hybrids were carried out at an accelerating voltage of $1 \mathrm{keV}$.

\section{Confocal laser scanning microscopy}

Immediately after the in situ preparation of C-dots within the porous structure, the samples were scanned with an LSM 700 confocal laser scanning microscope (CLSM; Carl Zeiss) connected to a Zeiss inverted microscope equipped with a Zeiss $\times 63$ oil immersion objective. Combinations of 405- and 555-nm laser lines were used to excite the $\mathrm{PSiO}_{2}$ structure and the C-dots, respectively. To obtain a three-dimensional projection of the porous structure, $z$-scans in $0.3 \mu \mathrm{m}$ increments were taken over a depth of $\sim 8 \mu \mathrm{m}$ and projected by using the ZEN 2009 (Carl Zeiss). Image analysis was performed with Imaris software (Bitplane AG, Zurich, Switzerland).

\section{Measurement of fluorescence emission spectra}

The fluorescence emission spectra of the $\mathrm{PSiO}_{2} / \mathrm{C}$-dot hybrids at different excitation wavelengths were recorded on a FL920 spectrofluorimeter (Edinburgh Instruments, Livingston, UK). For the trypsin- and ATP-sensing experiments, $100 \mu \mathrm{l}$ of trypsin or ATP at different concentrations (4.3-43 $\mu \mathrm{M}$ and $0.1-10 \mathrm{~mm}$, respectively) were employed. After incubation of the target analyte with the $\mathrm{PSiO}_{2} / \mathrm{C}$-dot hybrid for $1 \mathrm{~h}$, the emission spectra were measured at an excitation wavelength of $475 \mathrm{~nm}$. All sensing experiments were conducted in triplicate for each concentration.

\section{Transmission electron microscopy}

High-resolution transmission electron microscopy (HRTEM) studies of 'free' (prepared in solution) and confined (extracted from the hybrids) C-dots were carried out using a JEOL JEM-2100F HRTEM at an accelerating voltage of $200 \mathrm{keV}$. The transmission electron microscopy (TEM) studies of 'free' C-dots (prepared in solution) were carried out on an FEI Tecnai 12 G2 TWIN TEM at an accelerating voltage of $120 \mathrm{keV}$. The confined C-dot samples were prepared for observation by dissolving the $\mathrm{PSiO}_{2}$ matrix in a 3:1 (v/v) $\mathrm{HF}(48 \%)$ :ethanol (99.9\%) solution, followed by extraction of the C-dots from the matrix. Next, the C-dot solution (in absolute ethanol) was dropped on a graphene-coated copper grid.

\section{Fourier transform infrared spectroscopy}

Fourier transform infrared (FTIR) spectra of the C-dots synthesized in the $\mathrm{PSiO}_{2} / \mathrm{C}$-dot matrix and in solution were recorded on a Thermo Scientific Nicolet 6700 FTIR spectrometer by application of few mg of dry sample.

\section{X-ray photoelectron spectroscopy}

X-ray photoelectron spectroscopy (XPS) was performed using an X-ray photoelectron spectrometer ESCALAB 250 ultrahigh vacuum $\left(1 \times 10^{-9}\right.$ bar $)$ apparatus with an $\mathrm{AlK}^{\alpha} \mathrm{X}$-ray source and a monochromator. The X-ray beam size was $500 \mu \mathrm{m}$, and survey spectra were recorded with a pass energy (PE) of $150 \mathrm{eV}$; high-energy resolution spectra were recorded with a PE of $20 \mathrm{eV}$. Processing of the XPS results was carried out using Avantage software (Thermo Scientific, East Grinstead, UK).

\section{Measurement of interferometric reflectance spectra}

Interferometric reflectance spectra of the samples were collected using a charge-coupled device (CCD) spectrometer (USB4000, Ocean Optics, Largo, FL, USA) fitted with a microscope objective lens coupled with a bifurcated fiber optic cable. A tungsten light source was focused onto the center of the sample 


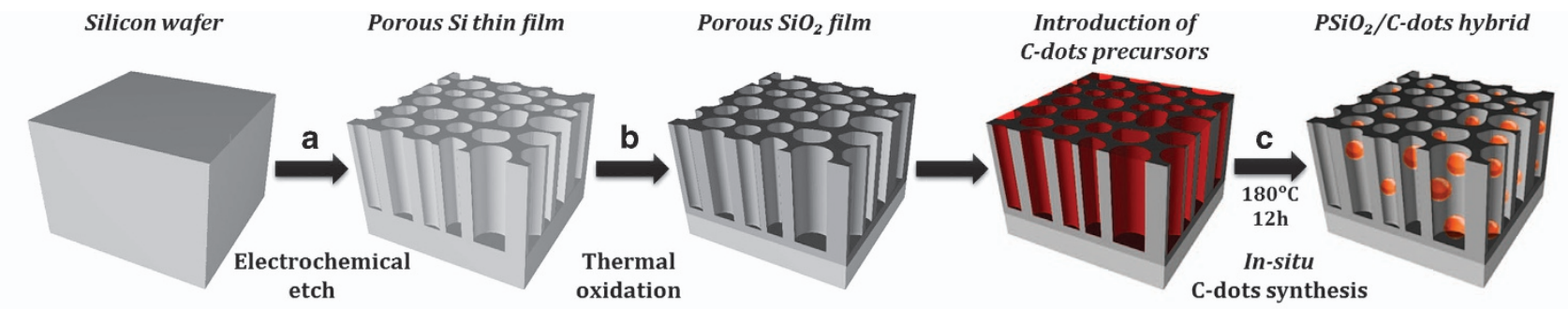

d

Reflectivity spectrum of $\mathrm{PSiO}_{2} / \mathrm{C}$-dots hybrid:

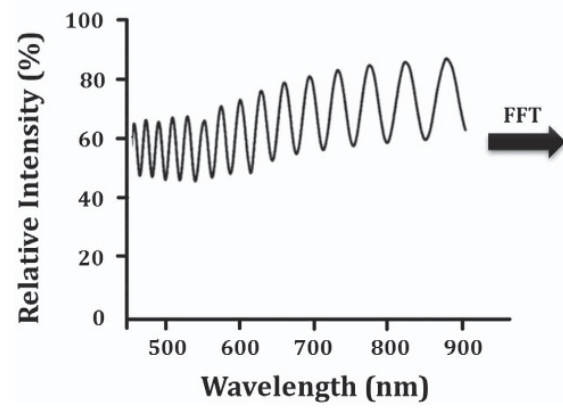

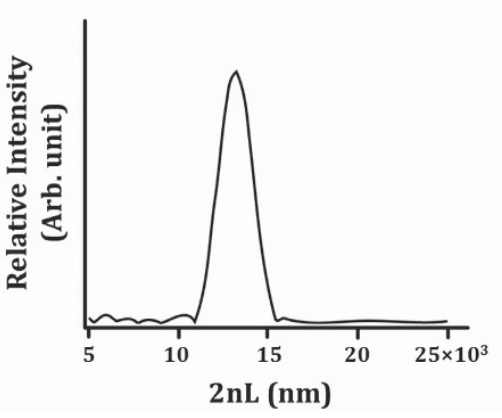

e

Fluorescence signal of $\mathrm{PSiO}_{2} / \mathrm{C}$-dots hybrid:

Figure 1 Construction of the C-dot/PSiO 2 hybrid and the two sensing modalities. (a) Anodic electrochemical etching is used to prepare a PSi layer from a single-crystal $\mathrm{Si}$ wafer; (b) the freshly etched sample is thermally oxidized at $800{ }^{\circ} \mathrm{C}$; (c) the carbonaceous precursor is incorporated in the $\mathrm{PSiO}_{2}$ pores for the in situ synthesis of the C-dots. Subsequent water evaporation and slow heating results in the formation of C-dots. (d) Optical reflectivity and (e) fluorescence emission generated simultaneously in the $\mathrm{C}$-dot/ $\mathrm{PSiO}_{2}$ hybrid. $\mathrm{C}$-dot, carbon dot; $\mathrm{PSiO}_{2}$, porous silicon oxide.

surface with a spot size of $\sim 1-2 \mathrm{~mm}^{2}$. Reflectivity data were recorded in a wavelength range of 500-900 $\mathrm{nm}$ with a spectral acquisition time of $100 \mathrm{~ms}$. Both illumination of the surface and detection of the reflected light were performed along an axis coincident with the surface normal. All the optical experiments were conducted in a fixed cell to assure that the sample reflectivity was measured at the same spot during all measurements. Spectra were collected in real time and analyzed by applying fast Fourier transform. The optical data in this paper are presented as relative $\triangle \mathrm{EOT}$, and $\Delta \mathrm{EOT}(t) / \mathrm{EOT}_{0}=\left(\mathrm{EOT}(t)-\mathrm{EOT}_{0}\right) / \mathrm{EOT}_{0}$, where the term $\mathrm{EOT}_{0}$ refers to the averaged effective optical thickness obtained during baseline establishment at the beginning of the optical experiments. All sensing experiments were conducted in triplicate for each concentration of trypsin or ATP.

Interferometric reflectance spectral measurements and fluorescence imaging—simultaneous bimodal sensing

To collect both the interferometric reflectance spectra and the fluorescence signal, we used a customized Zeiss upright microscope equipped with an Ocean Optics CCD USB 4000 spectrometer. A two-port adapter was utilized to selectively transmit the light either to the collimator, which was coupled to a fiber optic cable, or to the microscope camera (Axio Cam MRc, Zeiss). Supplementary Figure S12 (Supporting Information) depicts an image of this experimental set-up. The $\mathrm{PSiO}_{2} / \mathrm{C}$-dot hybrid was fixed to the microscope stage under the microscope objective. The light from a halogen source was focused through the microscope objective, and the size of the illumination spot was controlled with the iris of the microscope. Both the illumination of the surface and the detection of the reflected light were performed perpendicular to the surface. Optical reflectivity spectra were collected using the CCD spectrometer and analyzed by applying fast Fourier transform, as previously described. Fluorescence imaging was carried out concurrently to the reflectivity measurements at a constant exposure time of $100 \mathrm{~ms}$ using the camera.

\section{Statistical analysis}

Statistical analysis was performed using Student's $t$-test with a minimum confidence level of 0.05 for statistical significance, assuming unequal sample sizes and variance. All values are reported as the mean and the s.d. of the mean.
The detection limits were calculated from the linear correlation plot using $3 S_{\mathrm{e}} / m$, where $S_{\mathrm{e}}$ is the s.e. and $m$ is the slope.

\section{RESULTS AND DISCUSSION}

\section{Synthesis of the $\mathrm{PSiO}_{2} / \mathrm{C}$-dot hybrid}

Figure 1 depicts the synthetic scheme for the generation of the $\mathrm{PSiO}_{2} / \mathrm{C}$-dot hybrid and the bimodal sensing achieved with the material. We began with the anodization of a heavily doped p-type crystalline Si wafer (Figure 1a), which, after dry thermal oxidation, yielded a highly porous $\mathrm{SiO}_{2}$ film (Figure 1b). Subsequent incorporation of the carbonaceous precursor and pyrolysis produced the hybrid material, in which fluorescent C-dots were embedded within the $\mathrm{PSiO}_{2}$ pores (Figure 1c). Figure 1d and e illustrate the orthogonal sensing modes of the $\mathrm{PSiO}_{2} / \mathrm{C}$-dot hybrid. Specifically, Figure $1 \mathrm{~d}$ shows a typical reflectivity spectrum (left) comprising a series of Fabry-Pérot interference fringes resulting from reflections at the top and bottom interfaces of the porous thin film. ${ }^{29}$ The maxima of these fringes are governed by the following relationship (Fabry-Pérot equation): ${ }^{29}$

$$
m \lambda=2 n L
$$

where $m$ is an integer, $n$ is the average refractive index, $L$ is the thickness of the porous film and $\lambda$ is the wavelength of the incident light. The $2 n L$ term in Eq. 1 is referred to as the EOT. A change in the average refractive index $(n)$ leads to a shift in the observed reflectivity spectrum that is correlated with changes in the EOT. The incorporation and accumulation of molecules within a porous nanostructure generally result in redshifts of the EOT due to the increase in the average refractive index of the thin film layer. ${ }^{13}$ Application of fast Fourier transform to the reflectivity data yielded a peak with characteristic EOT and intensity (Figure 1d, right), which are distinctive features of the detected analytes. ${ }^{30,31}$ Figure 1e depicts a representative fluorescence emission spectrum generated by the C-dots embedded within the $\mathrm{PSiO}_{2}$ pores. Modulation of the fluorescence of 

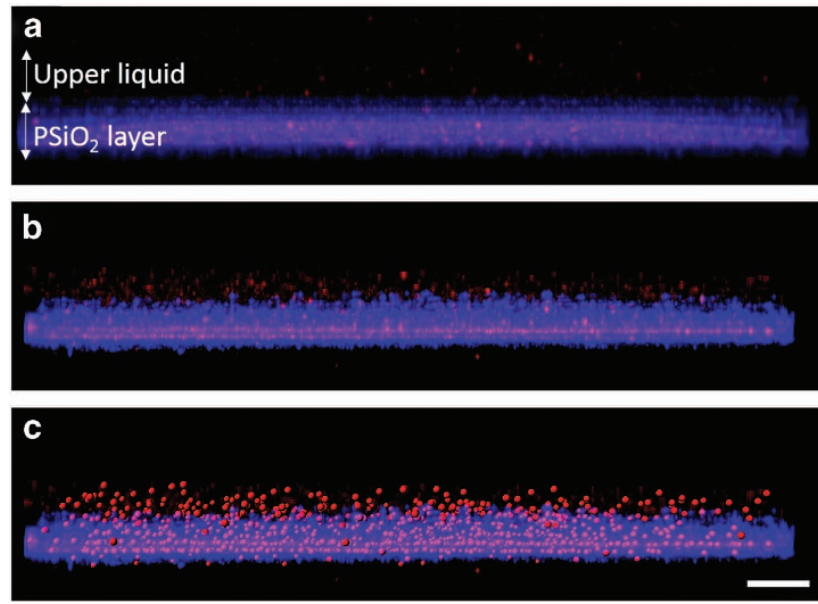

Figure $2 \mathrm{CLSM} 3 \mathrm{D}$ projection images of (a) neat $\mathrm{PSiO}_{2}$, (b) the $\mathrm{C}$-dot/ $/ \mathrm{PSiO}_{2}$ hybrid and (c) the $\mathrm{C}$-dot/ $\mathrm{PSiO}_{2}$ hybrid in which the fluorescence signal of the $\mathbf{C}$-dots is falsely colored red to aid observation. Panels ( $\mathbf{b}$ and $\mathbf{c}$ ) demonstrate the location of the C-dots within the porous layer in the $z$-direction. The scale bar is $3 \mu \mathrm{m}$. Shown are the net photoluminescence and fluorescence signals (after background subtraction). C-dot, carbon dot; CLSM, confocal laser scanning microscopy; $\mathrm{PSiO}_{2}$, porous silicon oxide; 3D, three-dimensional.

the C-dots, that is, both the wavelength and intensity, is induced upon adsorption of molecules in close proximity to the embedded carbon nanoparticles. $^{32}$

\section{Characterization of the $\mathrm{PSiO}_{2} / \mathrm{C}$-dot hybrids}

Figures 2 and 3 depict the microscopic analyses of the $\mathrm{PSiO}_{2} / \mathrm{C}$-dot hybrid, which were designed to probe the structural features of the material. To validate the occurrence of in situ synthesis of C-dots within the $\mathrm{PSiO}_{2}$ nanostructure, the hybrid was examined with CLSM (Figure 2). Figure 2 clearly reveals the dispersion of C-dots within the $\mathrm{PSiO}_{2}$ pores. Specifically, the blue photoluminescence corresponding to the $\mathrm{PSiO}_{2}$ scaffold $\left(\lambda_{\mathrm{ex}}=405 \mathrm{~nm}, \lambda_{\mathrm{em}} \geq 420 \mathrm{~nm} \text {, long pass filter }\right)^{33}$ allows for the analysis of the host matrix (Figure 2a) and can be spatially correlated to the fluorescence of the red C-dots $\left(\lambda_{\mathrm{ex}}=555 \mathrm{~nm}\right.$, $\lambda_{\mathrm{em}} \geq 560 \mathrm{~nm}$, long pass filter) within the porous layer (Figure $2 \mathrm{~b}$ ). Indeed, the fluorescence of the C-dots was observed throughout the entire depth of the porous scaffold over a distance of $\sim 4 \mu \mathrm{m}$. Residual fluorescence signals of the C-dots were also detected in a region slightly above the $\mathrm{PSiO}_{2}$ interface (Figure $2 \mathrm{~b}$ and c), likely because of attachment of the particles to the $\mathrm{PSiO}_{2}$ surface. To confirm that the C-dots did not leach out of the $\mathrm{PSiO}_{2}$ layer, the hybrid was incubated in a buffer solution for several hours, and the optical reflectivity spectrum was continuously monitored. Indeed, no baseline drift was observed in these experiments (Supplementary Figure S1, Supporting Information), confirming the stability and retention of the encapsulated C-dots within the $\mathrm{PSiO}_{2}$ host matrix.

The microscopy analyses presented in Figure 3 reveal the morphologies of both the $\mathrm{PSiO}_{2}$ matrix and the embedded C-dots. The HRSEM image of a cross-section of the untreated $\mathrm{PSiO}_{2}$ provided in Figure $3 \mathrm{a}$ shows the typical aligned pore structure of the $\mathrm{PSiO}_{2}$ matrix. ${ }^{28}$ The comparative HRSEM micrographs of the $\mathrm{PSiO}_{2} / \mathrm{C}$-dot hybrid shown in Figure $3 \mathrm{~b}$ and $\mathrm{c}$ reveal the nanostructure of the hybrid and the presence of organic material throughout the porous scaffold. ${ }^{34}$ Moreover, electron-charging effects were encountered throughout the entire cross-sections shown in Figure $3 \mathrm{~b}$ and $\mathrm{c}$, which was ascribed to the abundant C-dots-an organic substance-within the $\mathrm{PSiO}_{2}$ pores. ${ }^{35,36}$

The TEM and HRTEM images shown in Figure $3 \mathrm{~d}$ and e, respectively, reveal the structural features of the C-dots prepared in situ within the nanoscale pores. The C-dots extracted from the $\mathrm{PSiO}_{2}$ matrix by dissolving the porous host in hydrofluoric acid appear uniform (see Figure 3d; the size distribution analysis indicates a value of $3.7 \pm 0.6 \mathrm{~nm}$, Supplementary Figure S2, Supporting Information). A representative HRTEM image of the collected nanoparticles (Figure $3 \mathrm{e}$ ) highlights the crystalline graphite cores of the in situ synthesized C-dots. It should be noted that the 'free' C-dots prepared in solution were significantly larger (average diameter of $\sim 10 \mathrm{~nm}$, see Supplementary Figure S3, Supporting Information) than the confined C-dots synthesized in situ within the porous nanostructure. Thus, these results indicate that the C-dots formed under nanoscale confinement of the carbonaceous precursor within the $\mathrm{PSiO}_{2}$ host differed in their nanostructures and thus in their physicochemical properties relative to the C-dots synthesized in solution.

Figure 4 presents the spectroscopic characterization of the C-dots formed through the synthetic scheme outlined in Figure 1 in comparison with the C-dots prepared in solution from the same carbonaceous precursor. The FTIR data shown in Figure 4a show the expected surface functional groups, which are apparent in both C-dot samples. Specifically, Figure $4 \mathrm{a}$ reveals FTIR bands corresponding to $\mathrm{O}-\mathrm{H}$ and $\mathrm{N}-\mathrm{H}$ stretching ${ }^{4,7}\left(3330 \mathrm{~cm}^{-1}\right)$, the $\mathrm{C}=\mathrm{O}$ band of carbonyl and amide groups $\left(1690 \mathrm{~cm}^{-1}\right), \mathrm{C}=\mathrm{C}$ stretching $/ \mathrm{N}-\mathrm{H}$ bending $\left(1620 \mathrm{~cm}^{-1}\right)$ and amide II and amide I groups $\left(1500\right.$ and $1300 \mathrm{~cm}^{-1}$, respectively). Note that the peak positions exactly coincide in the two C-dot samples, indicating their similar C-dot surface structures; the differences in some of the peak intensities are consistent with the distinct synthetic environments of the two C-dot samples, that is, within the $\mathrm{PSiO}_{2}$ matrix (i) and in solution (ii).

The XPS data shown in Figure $4 \mathrm{~b}$ depict the atomic species present in the C-dots and further highlight the differences between C-dots prepared in situ from the confined precursor within the $\mathrm{PSiO}_{2}$ matrix and the C-dots synthesized in solution. Like the FTIR data shown in Figure $4 \mathrm{a}$, the same XPS C $1 \mathrm{~s}, \mathrm{O} 1 \mathrm{~s}$ and $\mathrm{N} 1 \mathrm{~s}$ signature peak positions were observed for the C-dots extracted from the $\mathrm{PSiO}_{2} / \mathrm{C}$ dot hybrids (Figure 4bi) and for the C-dots synthesized in solution (Figure 4bii). Specifically, the deconvoluted C $1 s$ spectrum displays peaks at $284.6 \mathrm{eV}$, corresponding to $s p^{2}$ carbon atoms $(\mathrm{C}=\mathrm{C})$, $285.8 \mathrm{eV}$, assigned to $\mathrm{C}-\mathrm{OH}$ groups and $287.5 \mathrm{eV}$, for $-\mathrm{COOH}$ and/or -COOR groups. ${ }^{4,7}$ The $\mathrm{O} 1 \mathrm{~s}$ spectrum shows peaks at $531.3 \mathrm{eV}$ for $\mathrm{C}=\mathrm{O}$ groups and $532.5 \mathrm{eV}$ for $\mathrm{O}=\mathrm{C}=\mathrm{OH}$ and/or $\mathrm{C}-\mathrm{OH}$ groups, and the $\mathrm{N} 1 s$ data present a peak at $399.2 \mathrm{eV}$ for $\mathrm{C}-\mathrm{N}$ and/or $\mathrm{C}=\mathrm{N}$ groups and a signal at $400.5 \mathrm{eV}$ corresponding to an $\mathrm{N}-\mathrm{O}$ group (Figure $4 \mathrm{~b}$ ). Importantly, Figure $4 \mathrm{~b}$ demonstrates the different deconvoluted XPS peak intensities for the C-dots prepared in situ inside the $\mathrm{PSiO}_{2}$ matrix, confirming that distinctive C-dots were formed within the nanopores.

The excitation-dependent fluorescence emission spectra of the C-dots extracted from the $\mathrm{PSiO}_{2}$ matrix and synthesized in solution are depicted in Figure 4c. Interestingly, both the shifts and relative intensities of the excitation-dependent emission peaks differed between the two C-dot samples. The relative intensities of the excitation-dependent spectra, however, were dependent on the synthetic scheme. In particular, the peaks of the C-dots prepared within the nanoscale pores (Figure $4 \mathrm{c}-\mathrm{i}$ ) were blueshifted compared with those of the C-dots prepared from the solution-dissolved precursor (Figure 4cii). This fluorescence spectral shift is consistent 

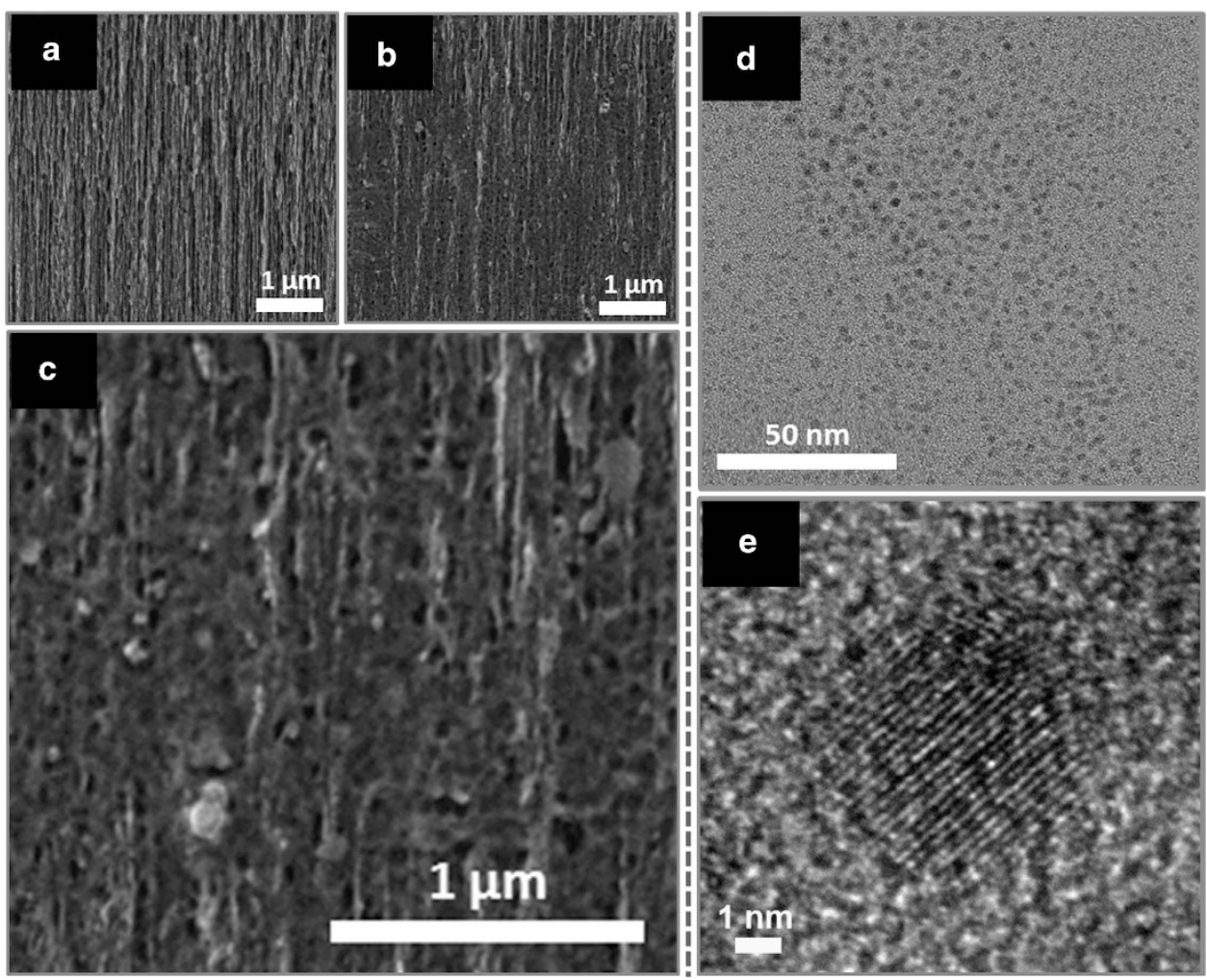

Figure 3 Microscopic characterization of the in situ synthesized C-dots. Cross-sectional HRSEM images (secondary electrons) of (a) the neat PSiO 2 layer (etched for $30 \mathrm{~s}$ at $300 \mathrm{~mA} \mathrm{~cm}{ }^{-2}$ ); (b) the $\mathrm{C}$-dot/PSiO ${ }_{2}$ hybrid; (c) and the $\mathrm{C}$-dot/PSiO ${ }_{2}$ hybrid at a higher magnification, showing the carbonaceous materials (that is, C-dots) interspersed within the pores. (d) TEM and (e) HRTEM images of the C-dots extracted from the PSiO 2 host matrix. C-dot, carbon dot; HRSEM, high-resolution scanning electron microscopy; HRTEM, high-resolution TEM; $\mathrm{PSiO}_{2}$, porous silicon oxide; TEM, transmission electron microscopy.

with the FTIR and XPS data presented in Figure $4 \mathrm{a}$ and $\mathrm{b}$ and is ascribed to slight differences in the surface characteristics of the C-dots resulting from their synthetic conditions and environment. Ultraviolet-visible (UV-vis) absorbance spectra of the two samples (Supplementary Figure S4, Supporting Information) reveal distinct spectral shifts and extinction coefficients for the two C-dots, further highlighting the different structural features of the two types of carbon nanoparticles. To further confirm that the fluorescence signal of the hybrid originates from the C-dots and not from the $\mathrm{PSiO}_{2}$ nanostructure, the photoluminescence spectra of neat $\mathrm{PSiO}_{2}$ was measured (Supplementary Figure S8, Supporting Information). A very weak photoluminescence signal was recorded from the $\mathrm{PSiO}_{2}$ matrix, confirming that the fluorescence signal of the hybrid was derived from the C-dots present within the nanostructured host.

\section{Bimodal detection of trypsin and ATP}

To investigate the application of the new $\mathrm{PSiO}_{2} / \mathrm{C}$-dot hybrid as a bimodal sensor for biomolecular analytes, we tested its optical and fluorescence responses to trypsin (a model protein target) and ATP (a model small analyte). Trypsin is a digestive enzyme produced in the pancreas as the proenzyme trypsinogen, and its concentration in the human body is correlated to pancreatic diseases, for example, pancreatic cancer and inflammation. ${ }^{37,38}$ ATP is known as the basic biological energy source of life. In addition, ATP is a marker for cell viability as it is present in all metabolically active cells, and its concentration may change in the case of disease..$^{39,40}$

Figure $5 \mathrm{a}$ depicts the relative EOT changes of the $\mathrm{PSiO}_{2} / \mathrm{C}$-dot hybrid during exposure to different concentrations of trypsin. Specifically, the infiltration of trypsin was monitored in real time by acquisition of the reflectivity spectra of the porous film and the normalized EOT. Notably, Figure 5a shows a rapid increase of $\sim 0.1$, 0.25 and $0.65 \%$ in the EOT in the presence of increasing concentrations of trypsin $(4.3,21.5$ and $43 \mu \mathrm{M}$, respectively), which is attributed to the infiltration and accumulation of trypsin in the nanostructured pores. A subsequent gradual increase was observed until the EOT values became stable (Figure 5aii), corresponding to saturated levels of the infiltrated protein. Control experiments with only buffer (no trypsin) produced no change in the EOT (Figure 5a, orange trace).

The bar diagram provided in Supplementary Figure S5 (Supporting Information) displays the maximal EOT values recorded upon the addition of different trypsin concentrations to the $\mathrm{PSiO}_{2} / \mathrm{C}$-dot hybrid. The reflectivity response was found to be proportional to the trypsin concentration, and a linear correlation was demonstrated $\left(R^{2}=0.95\right)$. Control experiments with neat $\mathrm{PSiO}_{2}$ (no C-dots) produced smaller changes in the EOT (Supplementary Figure S6, Supporting Information), a higher signal to noise ratio and a weaker linear correlation $\left(R^{2}=0.75\right)$ relative to the hybrid system. Thus, these results indicate that the presence of the C-dots within the nanostructure improved the sensitivity of the thin film through refractive index changes. The larger shifts observed in the hybrid system are ascribed to the presence of C-dots within the $\mathrm{PSiO}_{2}$ host, which reduces the nanoscale pore diameter and enhances the sensitivity of the thin film structure to refractive index changes. ${ }^{41,42}$ Importantly, the sensitivity of the $\mathrm{PSiO}_{2} / \mathrm{C}$-dot sensor, with a calculated limit of detection of $\sim 4 \mu \mathrm{M}$, is comparable to those of previously reported $\mathrm{PSiO}_{2}$ optical biosensors. ${ }^{43}$ Supplementary Table S1 (Supporting Information) summarizes the trypsin-sensing performance of the hybrid system in comparison with that of the control systems (for example, neat $\mathrm{PSiO}_{2}$ and 'free' $\mathrm{C}$-dots). 
a

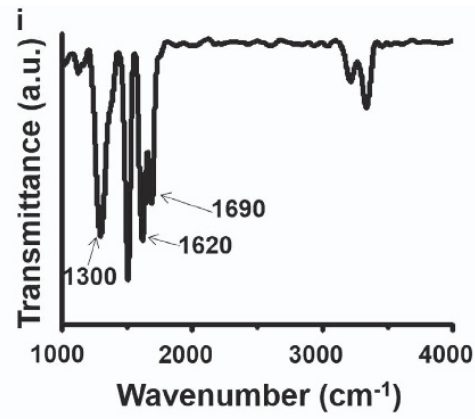

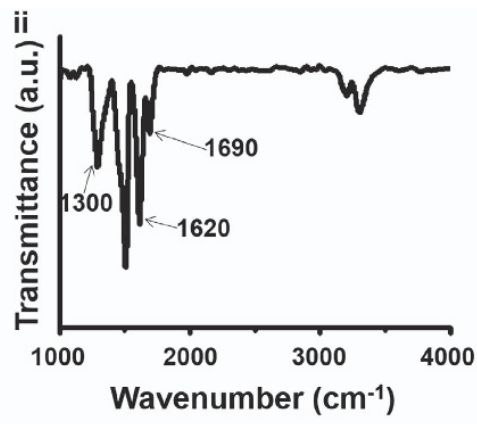

b i
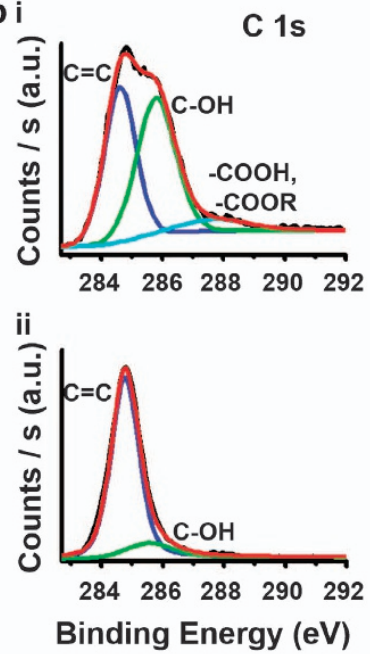
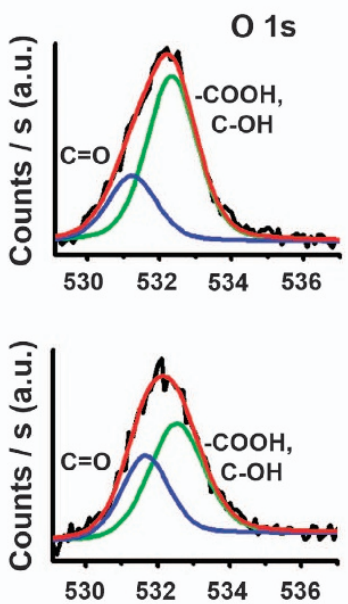

Binding Energy (eV)
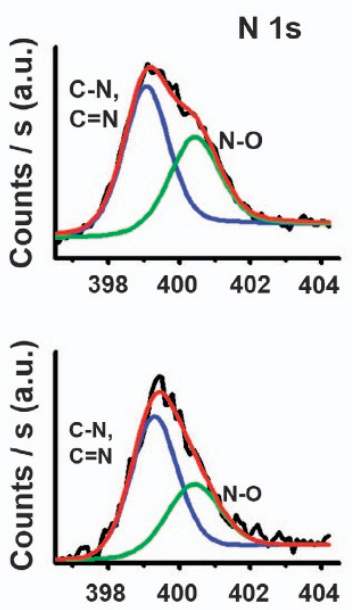

Binding Energy (eV)
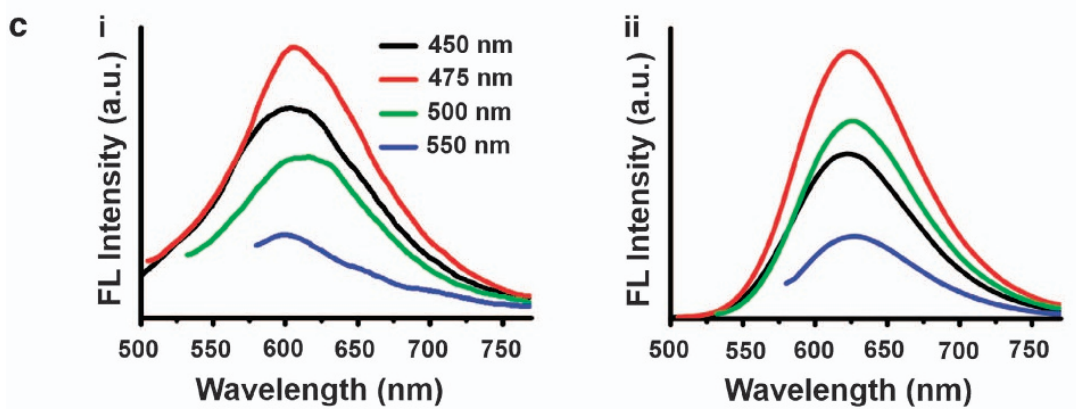

Figure 4 Characterization of the C-dots (i) extracted from the C-dot/PSiO 2 hybrid and (ii) synthesized in solution. (a) FTIR spectra; (b) XPS showing the different functional groups on the C-dot surface; and (c) fluorescence emission spectra from excitation at different wavelengths (indicated by the different colors). C-dot, carbon dot; FTIR, Fourier transform infrared; $\mathrm{PSiO}_{2}$, porous silicon oxide; XPS, X-ray photoelectron spectra.

It should be emphasized that conjugation of recognition elements or surface-displayed binding agents to the $\mathrm{PSiO}_{2} / \mathrm{C}$-dot hybrid would likely facilitate even greater sensitivity to the biomolecular targets. ${ }^{44}$

Figure $5 \mathrm{~b}$ presents the fluorescence emission spectra (excitation at $475 \mathrm{~nm}$ ) of the $\mathrm{PSiO}_{2} / \mathrm{C}$-dot hybrid upon incubation with different trypsin concentrations. Figure $5 \mathrm{~b}$ demonstrates a direct relationship between the trypsin concentration and quenching of the C-dot fluorescence. Moreover, the quenching of the C-dot fluorescence was found to be proportional to the trypsin concentration, and a linear correlation was demonstrated $\left(R^{2}=0.95\right.$, Supplementary Figure S7, Supporting Information). Quenching of the C-dot fluorescence by various analytes has been widely reported and is generally ascribed to electron and energy transfer between the target molecules and surface residues on the $\mathrm{C}$-dots, inducing non-radiative decay processes of the C-dot excitons. ${ }^{45}$ In the case of trypsin, the quenching of the fluorescence signal of the C-dots may be ascribed to $\pi-\pi$ interactions between the aromatic ring system of the C-dots and the hydrophobic units of trypsin. ${ }^{46,47}$ Notably, the 'free' C-dots prepared in solution exhibited an inferior degree of fluorescence quenching when exposed to trypsin (Supplementary Figure S9, Supporting Information) in comparison with the confined C-dots, underscoring the significance of the $\mathrm{PSiO}_{2}$ matrix in promoting the adsorption of the target analytes and their subsequent effect on the embedded C-dots.

To further investigate the applicability of the new $\mathrm{PSiO}_{2} / \mathrm{C}$-dot hybrid as a dual-mode analytical platform, we studied its optical and fluorescence responses to ATP, a model small analyte. Small molecules are difficult to detect by reflectivity, as the change in the average refractive index of the porous film is too low at relevant analyte concentrations. ${ }^{48}$ Indeed, RIFTS experiments employing different concentrations of ATP (Figure 5c) revealed a detection threshold of $5 \mathrm{mM}$, which is generally too high for practical sensing applications, and similar results were obtained for neat $\mathrm{PSiO}_{2}$ (as shown in 
a

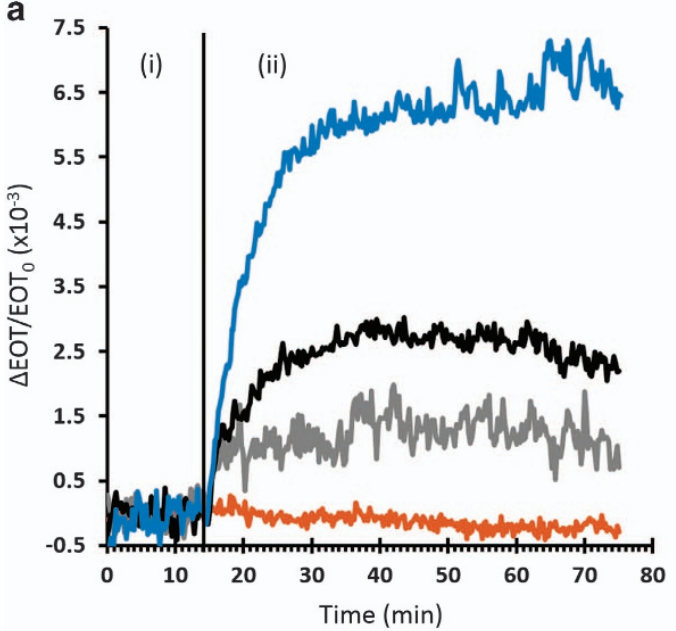

C

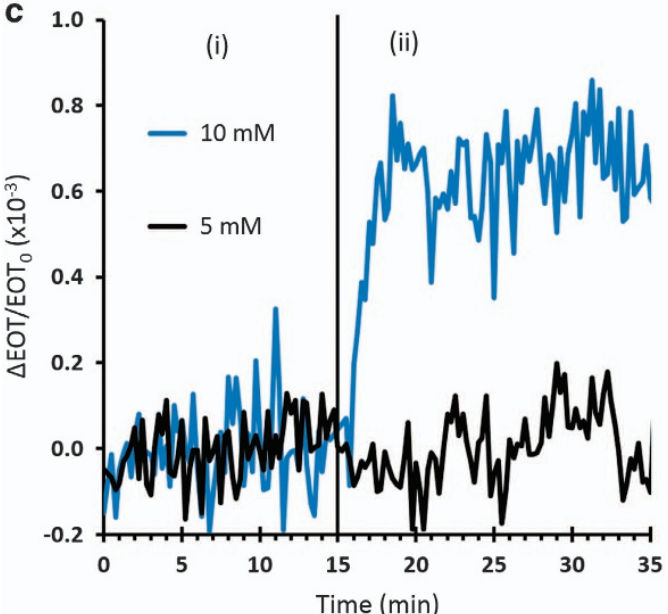

b

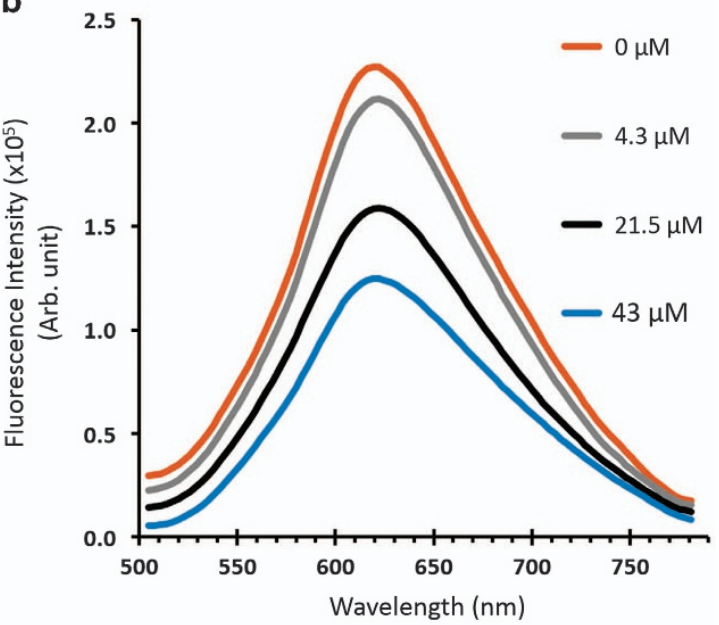

d

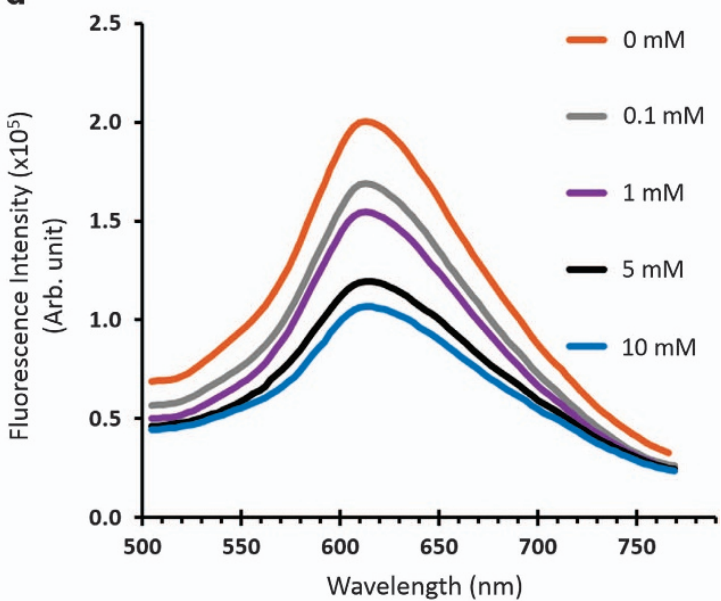

Figure 5 Bimodal sensing of trypsin (upper panel) and ATP (lower panel) by the C-dot/PSiO 2 hybrid. Optical responses to trypsin (a) and ATP (c), expressed in terms of the relative EOT of the $\mathrm{C}$-dot/PSiO 2 hybrid versus time of analyte infiltration into the nanostructure. (i) PBS buffer and (ii) introduction of the target analyte. The hybrid was fixed in a custom-made flow cell, and the reflectivity spectra were recorded every $15 \mathrm{~s}$. Fluorescence spectra of the C-dot/PSiO 2 hybrid (exc. $475 \mathrm{~nm}$ ) after incubation with trypsin (b) and ATP (d) at different concentrations. C-dot, carbon dot; EOT, effective optical thickness; PBS, phosphate-buffered saline; $\mathrm{PSiO}_{2}$, porous silicon oxide.

Supplementary Figure S10, Supporting Information). However, this drawback could be overcome by utilizing the fluorescence properties of the embedded C-dots. As apparent in the fluorescence spectra shown in Figure 5d, exposure to a low ATP concentration of $0.1 \mathrm{~mm}$ resulted in a decrease in the fluorescence signal of the C-dots. Figure $5 \mathrm{~d}$ also illustrates the occurrence of a direct relationship between the ATP concentration and the quenching of the C-dot fluorescence. It is suggested that the quenching of the C-dot fluorescence signal by the ATP molecules may be related to electron transfer from the nucleotides to the excited C-dots. ${ }^{49}$ Moreover, when comparing the sensing performance of the hybrid system to that of the 'free' C-dots (Supplementary Figure S11, Supporting Information), the quenching of the C-dot fluorescence in the hybrid system was found to be proportional to the ATP concentration, and a strong linear correlation was demonstrated $\left(R^{2}=0.98\right)$, whereas for the 'free' C-dots, a constant quenching effect was observed for high ATP concentrations $(\geq 1 \mathrm{~mm})$. Supplementary Table S2 (Supporting Information) summarizes the ATP-sensing performance of the hybrid system in comparison with that of the control systems (for example, neat $\mathrm{PSiO}_{2}$ and 'free' C-dots). Moreover, when comparing the sensitivity of the $\mathrm{PSiO}_{2} / \mathrm{C}$-dot sensor to ATP to that of previously reported QDs and carbon QD-based optical sensors, the limit of detection of the hybrid system is superior. ${ }^{49,50}$

While Figure 5 presents the optical and fluorescence signals recorded separately by different instrumentation, the $\mathrm{PSiO}_{2} / \mathrm{C}$-dot hybrid enables simultaneous orthogonal analyses of multiple adsorbed species. To demonstrate this capability, Figure 6 presents the optical reflectance data collected in parallel with the fluorescence emission upon placing the $\mathrm{PSiO}_{2} / \mathrm{C}$-dot hybrid under a fluorescence microscope equipped with a CCD spectrometer (the experimental set-up is shown in Supplementary Figure S12, Supporting Information). Figure 6 shows a small and slow change in the relative EOT prior to trypsin addition, whereas a rapid and significant increase of $\sim 0.55 \%$ in the EOT was clearly apparent upon introduction of trypsin $(43 \mu \mathrm{M})$, which was attributed to the infiltration and accumulation of the protein in the nanostructured pores. In parallel, the microscopy images shown in Figure 6 demonstrate that the fluorescent intensity of the hybrid was significantly quenched 20 min after the introduction of trypsin, consistent with the spectral data presented in Figure 4c. Overall, Figures 5 and 6 attest to the feasibility of bimodal sensing 


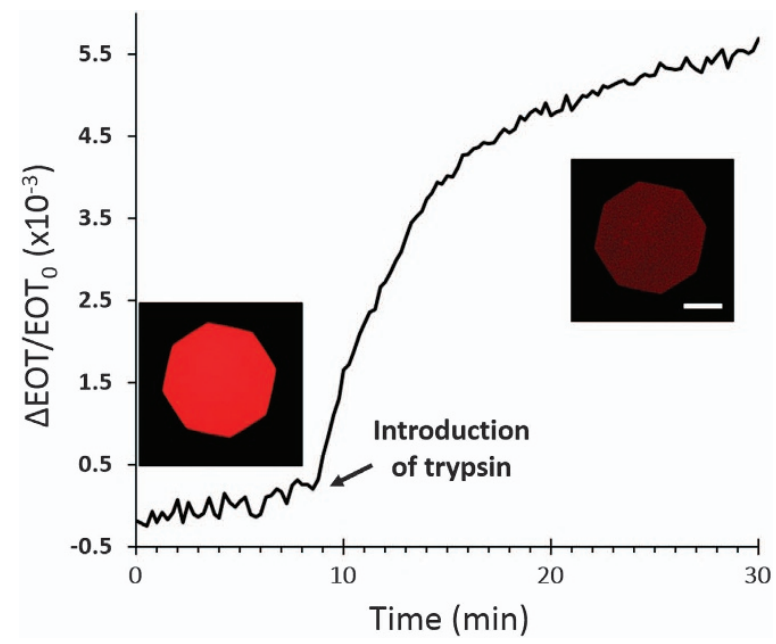

Figure $6 \mathrm{C}$-dot/PSiO 2 enables the simultaneous dual-mode detection of trypsin $(43 \mu \mathrm{m})$. Optical response expressed in terms of the relative EOT of $\mathrm{C}$-dot/PSiO ${ }_{2}$ versus exposure time to trypsin. The fluorescence microscopy image on the bottom left shows the matrix before the introduction of trypsin, whereas the image on the right corresponds to the $\mathrm{C}$-dot/ $\mathrm{PSiO}_{2}$ hybrid after incubation with trypsin for $20 \mathrm{~min}$. The scale bar corresponds to $100 \mu \mathrm{m}$. C-dot, carbon dot; $\mathrm{PSiO}_{2}$, porous silicon oxide.

through the simultaneous detection of trypsin and ATP by both optical reflectance of the $\mathrm{PSiO}_{2}$ matrix and quenching of the C-dot fluorescence. We are currently exploring several routes to enhance the selectivity of the hybrid sensor, including the use of appropriate surface chemistry and biofunctionalization of both components of the hybrid (that is, the $\mathrm{PSiO}_{2}$ nanostructure and the embedded C-dots) with specific capture probes (such as antibodies and aptamers). The suggested that a hybrid system reveals a new way of designing dualmode surface chemistry for two analytes simultaneously, as each of the hybrid elements can be modified with different capture probes.

\section{CONCLUSIONS}

We report the synthesis of a hybrid guest-host material comprising C-dots embedded within a $\mathrm{PSiO}_{2}$ matrix. To the best of our knowledge, this is the first report demonstrating the integration of C-dots within a nanoporous silicon host matrix. The preparation of the hybrid material was simple and involved incorporation of the C-dot carbonaceous precursor in the $\mathrm{PSiO}_{2}$ pores and subsequent heating. Spectroscopy and microscopy experiments confirmed the successful in situ synthesis of C-dots within the $\mathrm{PSiO}_{2}$ nanostructure host and showed that the $\mathrm{C}$-dots retained their unique fluorescence properties while being embedded within the $\mathrm{PSiO}_{2}$ pores. We demonstrated that the $\mathrm{PSiO}_{2} / \mathrm{C}$-dot hybrid enabled bimodal fluorescence/optical detection of different target analytes, including trypsin and ATP.

The potential of this hybrid system for modulating the photophysical properties of both components and for sensing applications is significant. The integration of $\mathrm{PSiO}_{2}$ and $\mathrm{C}$-dots enhances the sensing performance of these optically responsive materials in comparison with the individual components. Specifically, the $\mathrm{PSiO}_{2} / \mathrm{C}$-dot sensor exhibited both a significant increase in the effective refractive index of the porous layer and simultaneous quenching of the C-dot fluorescence signal upon addition of the analytes. Importantly, the C-dots embedded within the $\mathrm{PSiO}_{2}$ matrix exhibited a higher sensitivity than the solubilized C-dots, attesting to the significance of the nanoporous environment in promoting interactions between the carbon nanoparticles and adsorbed analytes and highlighting a useful strategy for enhancing the sensing capabilities of C-dots. Moreover, it should be emphasized that the hybrid allowed for real-time sensitive detection of small molecules, which is one of the main limitations of reflectivitybased PSi sensors. We are currently exploring several approaches for enhancing the sensitivity and selectivity of the reported hybrids, including the use of capture probes and changing the $\mathrm{PSiO}_{2}$ matrix from a single porous layer to a multilayered structure. Thus, the proofof-concept work presented here provides a generic sensing platform that can potentially be applied to the rapid detection and identification of a variety of analytes.

The orthogonal sensing modes of $\mathrm{PSiO}_{2} / \mathrm{C}$-dot provide a powerful method for sensing and identifying diverse analytes and are superior to the sensors based on the individual methods.

\section{CONFLICT OF INTEREST}

The authors declare no conflict of interest.

\section{ACKNOWLEDGEMENTS}

This work was supported by the German Research Foundation under grant SCHE 279/32, the Russell Berrie Nanotechnology Institute and the Lorry I. Lokey Interdisciplinary Center. Raz Jelink is grateful to the Ministry of Science and Technology Israel-China Program grant no. 3-13559. We thank Dr Nitzan Dahan from the LS\&E Infrastructure Unit at Technion for his valuable help with the confocal microcopy studies. Susanta Kumar Bhunia is thankful to the Planning and Budgeting Committee (PBC) of the Israeli Council for Higher Education for an Outstanding Postdoctoral Fellowship.

\section{PUBLISHER'S NOTE}

Springer Nature remains neutral with regard to jurisdictional claims in published maps and institutional affiliations.

1 Baker, S. N. \& Baker, G. A. Luminescent carbon nanodots: emergent nanolights. Angew. Chem. Int. Ed. 49, 6726-6744 (2010).

2 Lim, S. Y., Shen, W. \& Gao, Z. Q. Carbon quantum dots and their applications. Chem. Soc. Rev. 44, 362-381 (2015).

3 Zhu, S., Meng, Q., Wang, L., Zhang, J., Song, Y., Jin, H., Zhang, K., Sun, H., Wang, H. \& Yang, B. Highly photoluminescent carbon dots for multicolor patterning, sensors, and bioimaging. Angew. Chem. Int. Ed. 52, 3953-3957 (2013).

4 Bhunia, S. K., Maity, A. R., Nandi, S., Stepensky, D. \& Jelinek, R. Imaging cancer cells expressing the folate receptor with carbon dots produced from folic acid. Chembiochem 17, 614-619 (2016).

5 Ding, C. Q., Zhu, A. W. \& Tian, Y. Functional surface engineering of C-dots for fluorescent biosensing and in vivo bioimaging. Acc. Chem. Res. 47, 20-30 (2014).

6 Sun, X. \& Lei, Y. Fluorescent carbon dots and their sensing applications. Trends Anal. Chem. 89, 163-180 (2017).

7 Bhunia, S. K., Nandi, S., Shikler, R. \& Jelinek, R. Tuneable light-emitting carbon-dot/ polymer flexible films prepared through one-pot synthesis. Nanoscale $\mathbf{8}$, 3400-3406 (2016).

8 Dong, Y., Cai, J., You, X. \& Chi, Y. Sensing applications of luminescent carbon based dots. Analyst 140, 7468-7486 (2015).

9 Dong, Y., Li, G., Zhou, N., Wang, R., Chi, Y. \& Chen, G. Graphene quantum dot as a green and facile sensor for free chlorine in drinking water. Anal. Chem. 84, 8378-8382 (2012)

10 Dong, Y., Wang, R., Li, G., Chen, C., Chi, Y. \& Chen, G. Polyamine-functionalized carbon quantum dots as fluorescent probes for selective and sensitive detection of copper ions. Anal. Chem. 84, 6220-6224 (2012).

11 Lin, X., Gao, G., Zheng, L., Chi, Y. \& Chen, G. Encapsulation of strongly fluorescent carbon quantum dots in metal-organic frameworks for enhancing chemical sensing. Anal. Chem. 86, 1223-1228 (2014).

12 Wang, R., Li, G., Dong, Y., Chi, Y. \& Chen, G. Carbon quantum dot-functionalized aerogels for NO2 gas sensing. Anal. Chem. 85, 8065-8069 (2013).

13 Anglin, E. J., Cheng, L. Y., Freeman, W. R. \& Sailor, M. J. Porous silicon in drug delivery devices and materials. Adv. Drug Deliv. Rev. 60, 1266-1277 (2008).

14 Kilian, K. A., Boecking, T. \& Gooding, J. J. The importance of surface chemistry in mesoporous materials: lessons from porous silicon biosensors. Chem. Commun. 6 , 630-640 (2009).

15 Chen, M. Y., Klunk, M. D., Diep, V. M. \& Sailor, M. J. Electric-Field-assisted protein transport, capture, and interferometric sensing in carbonized porous silicon films. Adv. Mater. 23, 4537-4542 (2011). 
16 Massad-Ivanir, N., Segal, E. in Porous Silicon for Biomedical Applications (ed Santos, H. A.), 286-303 (Woodhead Publ Ltd, Abington Hall Abington, Cambridge Cb1 6ah, Cambs, UK, 2014).

17 Rodriguez, G. A., Ryckman, J. D., Jiao, Y., Fuller, R. L. \& Weiss, S. M. Real-time detection of small and large molecules using a porous silicon grating-coupled bloch surface wave label-free biosensor. Proc. SPIE 27, 8570 (2013).

18 Weiss, S. M., Rong, G. \& Lawrie, J. L. Current status and outlook for silicon-based optical biosensors. Phys. E 41, 1071-1075 (2009).

19 Guan, B., Magenau, A., Kilian, K. A., Ciampi, S., Gaus, K., Reece, P. J. \& Gooding, J. J. Mesoporous silicon photonic crystal microparticles: towards single-cell optical biosensors. Faraday Discuss. 149, 301-317 (2011).

20 Mariani, S., Pino, L., Strambini, L. M., Tedeschi, L. \& Barillaro, G. 10000-fold improvement in protein detection using nanostructured porous silicon interferometric aptasensors. ACS Sensors 1, 1471-1479 (2016).

21 Vilensky, R., Bercovici, M. \& Segal, E. Oxidized porous silicon nanostructures enabling electrokinetic transport for enhanced DNA detection. Adv. Funct. Mater. 25, 6725-6732 (2015)

22 Zhang, H., Jia, Z., Lv, X., Zhou, J., Chen, L., Liu, R. \& Ma, J. Porous silicon optical microcavity biosensor on silicon-on-insulator wafer for sensitive DNA detection. Biosensors Bioelectron. 44, 89-94 (2013).

23 Gaur, G., Koktysh, D. S. \& Weiss, S. M. Immobilization of quantum dots in nanostructured porous silicon films: characterizations and signal amplification for dual-mode optical biosensing. Adv. Funct. Mater. 23, 3604-3614 (2013).

24 Jiao, Y., Koktysh, D. S., Phambu, N. \& Weiss, S. M. Dual-mode sensing platform based on colloidal gold functionalized porous silicon. Appl. Phys. Lett. 97 (2010).

25 Krismastuti, F. S. H., Pace, S. \& Voelcker, N. H. Porous silicon resonant microcavity biosensor for matrix metalloproteinase detection. Adv. Funct. Mater. 24 3639-3650 (2014).

26 Liu, C., Jia, Z., Lv, X., Lv, C. \& Shi, F. Enhancement of QDs' fluorescence based on porous silicon Bragg mirror. Phys. B 457, 263-268 (2015)

27 Massad-Ivanir, N., Shtenberg, G., Zeidman, T. \& Segal, E. Construction and characterization of porous $\mathrm{SiO} 2 /$ hydrogel hybrids as optical biosensors for rapid detection of bacteria. Adv. Funct. Mater. 20, 2269-2277 (2010).

28 Urmann, K., Walter, J. G., Scheper, T. \& Segal, E. Label-free optical biosensors based on aptamer-functionalized porous silicon scaffolds. Anal. Chem. 87, 1999-2006 (2015).

29 Sailor, M. J. \& Link, J. R. "Smart dust": nanostructured devices in a grain of sand. Chem. Commun. 11, 1375-1383 (2005).

30 Pacholski, C., Sartor, M., Sailor, M. J., Cunin, F. \& Miskelly, G. M. Biosensing using porous silicon double-layer interferometers: reflective interferometric Fourier transform spectroscopy. J. Am. Chem. Soc. 127, 11636-11645 (2005).

31 Pacholski, C., Yu, C., Miskelly, G. M., Godin, D. \& Sailor, M. J. Reflective interferometric fourier transform spectroscopy: a self-compensating label-free immunosensor using double-layers of porous SiO2. J. Am. Chem. Soc. 128 4250-4252 (2006).

32 Wang, Y. \& Hu, A. Carbon quantum dots: synthesis, properties and applications. J. Mater. Chem. C 2, 6921-6939 (2014).

33 Sa'ar, A. Photoluminescence from silicon nanostructures: the mutual role of quantum confinement and surface chemistry. J. Nanophoton. 3, 42 (2009).

34 Massad-Ivanir, N., Friedman, T., Nahor, A., Eichler, S., Bonanno, L. M., Sa'ar, A. \& Segal, E. Hydrogels synthesized in electrochemically machined porous Si hosts: effect of nano-scale confinement on polymer properties. Soft Matter $\mathbf{8}$ 9166-9176 (2012).

35 Boubaya, M., Belhadj, A., Zangar, H. \& Blaise, G. An experimental study of the charging regime of the composite (PANI5\%/PMMA). J. Phys. D.Appl. Phys. 40, 4297-4303 (2007).
36 Butler, J. H., Joy, D. C., Bradley, G. F. \& Krause, S. J. Low-voltage scanning electronmicroscopy of polymer. Polymer 36, 1781-1790 (1995).

37 Hirota, M., Ohmuraya, M. \& Baba, H. The role of trypsin, trypsin inhibitor, and trypsin receptor in the onset and aggravation of pancreatitis. J. Gastroenterol. 41, 832-836 (2006).

38 Yamashita, K., Mimori, K., Inoue, H., Mori, M. \& Sidransky, D. A tumor-suppressive role for trypsin in human cancer progression. Cancer Res. 63, 6575-6578 (2003).

39 Zhang, X., Zhao, Y., Li, S. \& Zhang, S. Photoelectrochemical biosensor for detection of adenosine triphosphate in the extracts of cancer cells. Chem. Commun. 46, 9173-9175 (2010).

40 Zhou, Z.-M., Yu, Y. \& Zhao, Y.-D. A new strategy for the detection of adenosine triphosphate by aptamer/quantum dot biosensor based on chemiluminescence resonance energy transfer. Analyst 137, 4262-4266 (2012).

41 Ouyang, H., Striemer, C. C. \& Fauchet, P. M. Quantitative analysis of the sensitivity of porous silicon optical biosensors. Appl. Phys. Lett. 88, 3885-3894 (2006).

42 Rea, I., Sansone, L., Terracciano, M., De Stefano, L., Dardano, P., Giordano, M., Borriello, A. \& Casalino, M. Photoluminescence of graphene oxide infiltrated into mesoporous silicon. J. Phys. Chem. C 118, 27301-27307 (2014).

43 Shtenberg G., Segal E. in Handbook of Porous Silicon (ed Canham, L.), 857-868 (Springer International Publishing, Cham, 2014).

44 Zuo, J., Jiang, T., Zhao, X., Xiong, X., Xiao, S. \& Zhu, Z. Preparation and application of fluorescent carbon dots. J. Nanomater. 2015, 10-10 (2015)

45 Amjadi, M., Abolghasemi-Fakhri, Z. \& Hallaj, T. Carbon dots-silver nanoparticles fluorescence resonance energy transfer system as a novel turn-on fluorescent probe for selective determination of cysteine. J. Photochem. Photobiol. A 309 , 8-14 (2015).

46 Barati, A., Shamsipur, M. \& Abdollahi, H. Hemoglobin detection using carbon dots as a fluorescence probe. Biosensors Bioelectron. 71, 470-475 (2015).

47 Bui, T. T. \& Park, S. Y. A carbon dot-hemoglobin complex-based biosensor for cholesterol detection. Green Chem. 18, 4245-4253 (2016).

48 Krepker, M. A. \& Segal, E. Dual-functionalized porous Si/hydrogel hybrid for label-free biosensing of organophosphorus compounds. Anal. Chem. 85 7353-7360 (2013).

49 Callan, J. F., Mulrooney, R. C. \& Karnila, S. Luminescent detection of ATP in aqueous solution using positively charged CdSe-ZnS quantum dots. J. Fluorescence 18 , 1157-1161 (2008).

50 Gao, M. X., Liu, C. F., Wu, Z. L., Zeng, Q. L., Yang, X. X., Wu, W. B., Li, Y. F. \& Huang, C. Z. A surfactant-assisted redox hydrothermal route to prepare highly photoluminescent carbon quantum dots with aggregation-induced emission enhancement properties. Chem. Commun. 49, 8015-8017 (2013).

This work is licensed under a Creative Commons

Attribution 4.0 International License. The images or other third party material in this article are included in the article's Creative Commons license, unless indicated otherwise in the credit line; if the material is not included under the Creative Commons license, users will need to obtain permission from the license holder to reproduce the material. To view a copy of this license, visit http:// creativecommons.org/licenses/by/4.0/

C) The Author(s) 2018

Supplementary Information accompanies the paper on the NPG Asia Materials website (http://www.nature.com/am) 